\title{
Evaluator Roles and Strategies for Expanding Evaluation Process Influence
}

\author{
STEPHEN M. MORABITO
}

\begin{abstract}
This article explores various evaluator roles and strategies that have the potential to increase the likelihood that the evaluation process will have an influence on an organization and its members. These roles are (1) educator, (2) consultant, (3) facilitator, and (4) counselor. A brief case study presents the discussion dynamics of an evaluation workgroup. This workgroup resulted in programmatic changes based primarily on the discussion of developing indicators for measuring student outcomes. Practical implications for evaluators seeking to enhance process influence may involve the evaluator using a "client-centered" approach. Facilitating collaborative, open environments and managing discussion dynamics are fundamental in establishing a community of organizational learners. These dynamics may take the form of both task-focused and task-related discussions.
\end{abstract}

\section{EVALUATOR ROLES AND STRATEGIES FOR EXPANDING EVALUATION PROCESS INFLUENCE}

Process use has been described by Patton (1997, p. 88) as "ways in which being engaged in the process of evaluation can be useful quite apart from the findings that may emerge from those processes" (italics added for emphasis by Kirkhart, 2000, p. 10). Kirkhart (2000) presents the Integrated Theory of Evaluation Influence, which offers a new vocabulary for articulating the nuances of evaluation use and influence. "The term influence (the capacity or power of persons or things to produce effects on others by intangible or indirect means) is broader than use, creating a framework with which to examine effects that are multidirectional, incremental, unintentional, and noninstrumental, alongside those that are unidirectional, episodic, intended, and instrumental (which are well presented by the term use)" (Kirkhart, 2000, p. 7). In this article, the term "influence" is adopted in order to express the capacity of an evaluation process

Stephen M. Morabito - Research and Evaluation Center, National Center for Disability Services, 201 I.U. Willets Road, Albertson, NY 11507, USA; Tel: (1) 516-465-1607; Fax: (1) 516-747-3358; E-mail: smorabito@ ncds.org.

American Journal of Evaluation, Vol. 23, No. 3, 2002, pp. 321-330. All rights of reproduction in any form reserved. ISSN: 1098-2140 @ 2002 by American Evaluation Association. Published by Elsevier Science Inc. All rights reserved. 
to affect organizational stakeholders and the entity that is being evaluated. Furthermore, this language allows the field to categorize any organization-related change stimulated during the evaluation process as influence.

The roots and the evolution of process-based influence are particularly visible in the history of participatory evaluation models (Brisolara, 1998). The involvement of stakeholders contributes to process-based influence in that those involved in it shape the evaluation process. Stakeholders may show a level of enthusiasm for participatory evaluations unlike that of other evaluation methods. Preskill and Caracelli (1997) report that in a survey of American Evaluation Association members, "... the majority of respondents agreed that involving multiple stakeholders increases the use of evaluation processes (72\%)." However, it remains unclear as to what specific dynamics of that involvement would be most conducive to this influence. Moreover, how can an evaluator increase the likelihood that the evaluation process will have an influence on an organization?

Evaluators serve in a variety of roles to produce information that an organization will then use to make programmatic alterations. Whether an evaluation is summative or formative, there is certainly a strong consensus that the data and information gathered from the evaluation should be used in some way; in many cases, evaluators actively advocate for use. Utilization-focused evaluation (Patton, 1997) emphasizes promoting the use of the evaluation throughout the process. However, the influence of the evaluation process is many times an afterthought and seldom integrated into the goals of evaluation strategies. In addition, "the unintended influence of evaluation may be more impactful than intended influence" (Kirkhart, 2000, p. 12). Few evaluation methodologies incorporate a goal that seeks to achieve benefit from the evaluation process itself. An exception to this is the methodology articulated in Preskill and Torres (1999) in that it engages stakeholders in learning through the evaluative inquiry process. Preskill and Caracelli (1997) imply a need to examine further the relationship between organizational learning and process use, as well as understand the factors that contribute to or impede the extent to which organizational learning and process use evaluation approaches result in instrumental and conceptual use.

Although the specific evaluation methodology implemented within an organization may have a large effect on how the evaluation process influences an organization, this article focuses on the specific evaluator roles, philosophies, and interpersonal dynamics that hold the potential to foster a process influence. The true case study that follows is included as an example of an internal evaluator who has used various evaluator roles in interactions with organizational stakeholders. These roles and interactions contributed to the organization's willingness to make programmatic changes (influence) as a result of the evaluation process. The overall goal of the evaluation for the organization in the case study was to assess whether the actual student outcomes were consistent with the desired student outcomes. These desired outcomes were developed from the organization's vision and mission statements. To assist the organization in accomplishing this objective, the evaluator used a collaborative approach in which the knowledge and beliefs of organizational stakeholders are revered.

\section{Case Study}

A workgroup was held as part of an outcomes evaluation for a school that provides instruction to physically and medically disabled children from kindergarten through 12 th grade. The term "workgroup" is used in this context as a moderated gathering of organizational stakeholders to progress on a specific evaluation task. The goal of this workgroup was to develop 
indicators for already specified outcomes for students. The evaluator had advocated implementation of this process. Further, that it should include a range of school staff and administrators. As one of the major decision-makers, the superintendent's response was an enthusiastic "yes." There was general agreement that the involvement of faculty and staff was needed in the generation of outcomes indicators. In addition, all involved in this process were aware that the superintendent's approval and support was needed in implementing any evaluative methods or programmatic changes. The superintendent and other major decision-makers fully supported the evaluation and were committed to the improvement of services based on accurate, unbiased information.

In attendance at the workgroup were a variety of school administrators and staff, including two teachers, a social worker, a staff trainer, the assistant principal, the principal, the superintendent, and the evaluator/facilitator. The evaluator/facilitator had prepared a brief presentation that conveyed upper-level management's commitment to the evaluation and defined the task and key terms such as "outcome," "indicator," "data source," and "data collection method" (Hatry, van Houten, Plantz, \& Greenway, 1996). The objective, as presented to the workgroup participants, was "to use our knowledge and experience to define specific operations to evaluate outcomes for students." The evaluator/facilitator presented the task in this way to encourage participants to articulate their viewpoints.

One of the first discussions of this workgroup involved a debate about the data collection points. The objective at this stage (for evaluation purposes) was to promote a level of understanding about the defined data collection points (i.e., points in time when student outcome data would be collected) to facilitate the process of developing outcome indicators. In preliminary discussions with the superintendent before the workgroup, it was agreed upon that these data collection points would be 3rd, 6th, 8th, and 12th grades, and 1, 2, and 5 years post-graduation, which would coordinate with the State Assessment Standard structure as well as the school's "case conference teams." These teams discuss student progress (and outcomes) on a regular basis to provide additional support.

During the presentation of "data collection points," the group focused on other school issues, based upon some previous discussions among the school's administration, rather than on the task at hand-data collection points. These discussions related to the future possibility of restructuring the school to be consistent with the State Learning Standard structure as opposed to the State Assessment Standard structure. This new structure would essentially group students into elementary, middle, and high school categories.

The discussion of the new structure was tangential to the evaluation tasks at hand. However, given that the participants had an enthusiastic interest in this topic, the evaluator/facilitator encouraged the continuation of this discussion. It was important to agree on data collection points. However, the process had brought the staff to a juncture at which an immediate decision about a programmatic change could greatly enhance the ability to assess student outcomes later. Furthermore, problem-solving discussions of this nature are artifacts of the learning culture, which the evaluator encouraged even at the expense of immediate progress on the task at hand. After cautious progress, this task-related discussion was finalized with a commitment from the superintendent to restructure the school to parallel State Learning Standards. Essentially, the workgroup addressed the issues they felt were most important, and the discussion expedited the decision to implement structural changes.

At that point, the evaluator/facilitator encouraged a more task-focused discussion of the data collection points and how it would be appropriate to align them with the new structure for evaluative purposes. After deciding on data collection points, it was time for the group to 
focus on the task of developing outcome indicators using worksheets. The evaluator/facilitator provided some simple examples, such as:

Desired outcome: Students have enhanced communication skills;

Data collection point: Fourth grade;

Indicator: Demonstrated ability to dial 911.

It was then that the group deviated from the task by articulating various philosophical ideas of teaching, learning, and student support. Again, the evaluator/facilitator assisted the progress of this discussion despite the fact that progress toward the group's objective might decelerate. The essence of that debate was this: "If we are to assess our ability as a school to promote these student outcomes, then what can we do to improve outcomes for students now?" After lengthy discussion, the workgroup participants, with the support of the superintendent, decided to incorporate curriculum goals for each grade level that preceded a data collection point to enhance students' ability to achieve the desired outcomes. This task would be completed aside from the evaluation process, most likely within the school at case conference team meetings.

Indicators were developed in a relatively short period of time the next day. The workgroup met its objective and accomplished much more. The school is now in the process of setting the additional curriculum goals. Data are now being collected on the indicators that were formed into case conference team checklists.

\section{DISCUSSION}

The case study illustrates three major concrete changes that are indicative of a process influence: (1) the individual and team learning that occurred; (2) the addition of curriculum goals; and (3) the immediate decision to change the school structure. The evaluator in the case study was able to play various roles in his interaction with the workgroup participants. The main argument of this article is that these roles have the potential to foster an influential evaluation process.

Evaluator roles today are described by such terms as facilitator, problem solver, educator, coach, and critical friend (Caracelli, 2000). The evaluator played four roles in the case study that potentially increased the likelihood of an influential evaluation process: (1) educator, (2) consultant, (3) facilitator, and (4) counselor. In these roles, the evaluator manages the discussion dynamics of the group, encourages group learning, and essentially advocates that the evaluation process form the basis for programmatic change. In the traditional sense, an advocate promotes an idea by presenting relevant information in order to engage others to embrace that idea. In understanding the advocacy for an evaluation process influence, the advocate will serve in a variety of roles to open up opportunities for the evaluation to have an influence. Understanding and implementing the evaluator roles of educator, consultant, facilitator, and counselor have great potential to foster this influence.

\section{The Evaluator in the Role of Educator}

In the case study, the stakeholders were involved in the evaluation process, encouraged to discuss the issues that were most important to them, and engaged in a process of learning. "Process use refers to and is indicated by individual changes in thinking and behavior, and program or organizational changes in procedures and culture, that occur among those involved in evaluation as a result of the learning that occurs during the evaluation process" 
(Patton, 1997, p. 90). Learning, as defined by Catania (1992), is "a relatively permanent change in behavior based on experience" (p. 1). Therefore, process use and learning are very closely tied together, and the role of educator becomes an integral part of the evaluator's function in promoting an influential evaluation process.

It becomes clear that facilitating environments conducive to learning is an integral part of increasing the likelihood that the evaluation process will create change, whether at the organizational or individual level. Evaluators many times advocate for the use of findings and for the implementation of recommendations. Yet how does one advocate or promote an influential evaluation process? At the very least, the case study has shown that evaluators can advocate/promote an influential evaluation process by engaging in the role of educator. Specifically, the evaluator/facilitator in the case study engaged the members of the workgroup in the learning process of (a) dialogue, (b) reflection, (c) asking questions, and (d) identifying and clarifying values, beliefs, assumptions, and knowledge. Preskill and Torres (1999, p. 52) have labeled these activities as learning processes to communicate the spirit of evaluative inquiry. By fostering learning, the evaluator essentially becomes a promoter or advocate for process influence through the implementation of collaborative mechanisms by which stakeholders are free to share and learn. Preskill and Torres (1999) state that "Evaluative inquiry represents an emphasis on understanding each other in order to understand larger organizational challenges" (p. 2). On the most basic level, the creation of this community of learners involves contributing to ongoing organizational improvement through systematically gathering and sharing information (Sonnichsen, 2000). Again, learning is a change in behavior, whether it is the behavior of the organization or an individual. Logically, then, the evaluation process can have an influence on organizational stakeholders, and thus, the entity being evaluated, when the evaluator takes on the role of an educator and fosters learning.

\section{The Evaluator in the Role of Consultant}

Preskill (1994) illustrates how an evaluator might engage organizational members in creating individual and organizational learning by expanding on the traditional role of evaluator. She further reports that "This role definition requires the evaluator to become part of the change process by blending the role of organizational development consultant and evaluator" (p. 292). A consultant in this context refers to one who possesses a diverse collection of skills and knowledge with which to guide and advise the organization. "The key to successful internal consulting in organizations is to develop the image of the evaluation office as an organizational problem-solving asset with a diversity of knowledge and skills to employ in a variety of situations" (Sonnichsen, 2000, p. 168). This approach enhances the evaluator's ability to function as an agent of change and allows him/her to more fully incorporate their individual skills into their consultations with clients to promote this change. Providing this client-based service, internal evaluators seek to obtain a balance of independence and interdependence by ensuring that client viewpoints are heard while fostering an environment of organizational learning. As such, Sonnichsen (2000) states:

Internal evaluators in modern organizations will become more valuable to the organization if they see themselves and are seen by the organization as consultants with a broad range of practical, analytical skills rather than simply as evaluators. The roles of consultant, advocate, problem solver, evaluator, performance monitor, planner, facilitator, and management analyst combine to form a composite characterization of the modern, professional internal evaluator (p. 21). 
The internal evaluator, serving as the facilitator for the group activity in the case study, is philosophically grounded in an advocacy approach to evaluation. Advocacy evaluation is a philosophical and administrative approach to internal evaluation that seeks to increase the utility of internal evaluation information by emphasizing the role of evaluators as change agents (Sonnichsen, 2000). As a change agent, an evaluator focuses on tasks that he/she believes will ultimately promote discussion, debate, and positive organizational change based on his or her activities. In other words, the evaluator works toward being perceived as a consultant, an agent of change, and a contributor to ongoing organizational improvement. In this role, the evaluator has created an environment (the workgroup) with fundamental elements of synergy, empowerment, and learning. With a diverse set of knowledge and skills, the evaluator is looked upon as an individual who is there to guide the process. Therefore, the stakeholders take ownership of the process. This ownership translates into empowerment, and thus the process has the potential to have influential outcomes. The evaluator guides the organizational stakeholders by listening to what the issues are and by providing direction and guidance to resolve them. In the case study, this led to participant learning and programmatic changes. Therefore, the evaluator who serves as a consultant may be a critical factor in increasing the likelihood that the evaluation process will have an influence on clients.

\section{The Evaluator in the Role of Facilitator}

To facilitate is to ease, make possible, and smooth the progress of a process. With few exceptions, the primary goal of most evaluation processes is to make positive change possible. The argument against a facilitator role would certainly cite the importance of impartiality and objectivity within evaluation, such as the serious, systematic, and skilled methodologies advocated by Scriven (2001). Nonetheless, the way in which an evaluator facilitates groups of organizational stakeholders and the manner in which he/she interacts with them can itself contribute to the influence on the organization, as illustrated by the preceding case study.

With regard to facilitating groups, in general, Krueger (1998, p. 46) discusses the facilitator (moderator) as a seeker of wisdom: "[The] moderator is out to obtain understanding, insight and wisdom. [The] moderator assumes that the participants have that wisdom, and that, if asked the right questions, they will share it." Logically, people have the greatest understanding, insight, and wisdom about the issues that most excite them. For example, the participants in the workgroup genuinely cared for (and were most enthusiastic when discussing) students. The evaluator/facilitator encouraged the group to discuss the issues most important to them, even though this discussion was not an intended activity. By doing so, the process influenced the group such that their cohesion led to programmatic changes.

Focus groups, meetings, plenaries, and group processes that are fashioned to perform a specified objective (including open forums such as Open Space Technology, Harrison, 1997) can take the form of both task-focused discussion and task-related discussion. A task-focused discussion is one that is directly related to the desired outcome and purpose of the discussion. Task-related discussions are the more tangential conversations that evolve and may not be directly linked to the desired outcome or purpose but are certainly perceived to be related. The evaluator/facilitator in the case study encouraged the group to digress on the topics that were most interesting to them. As a facilitator, the evaluator was able to balance and maintain elements of both task-focused and task-related discussion dynamics.

The case study also indicates an evaluation process that had an unintended influence (see Kirkhart, 2000). By allowing the group to take on a task-related discussion, programmatic 
changes were made that could not be specifically predicted. As a matter of semantics, if we predict and desire unexpected outcomes (expecting the unexpected), does that imply that influence will be intended? This is a matter of perception and the stuff of philosophical debates. However, perhaps intentional influence can be specifically predicted beforehand, whereas unintended influence cannot. The point here is that an evaluator can create a group forum so that the evaluation process holds the potential to elicit unintended outcomes. Given this, a facilitator of a workgroup may be able to maintain a level of focus on the task at hand while encouraging an open forum where participants perceive they are at liberty to contribute those issues that are most important to them. The result, again, might be a quite influential evaluation process, whether the specific outcomes are intended or unintended.

\section{The Evaluator in the Role of Counselor}

There is a strong similarity between evaluating and counseling. This is especially true of evaluations designed to facilitate process influence, but may not be at all true of the more classic evaluation methodologies. An organization is a living collection of people, each with their own values, agendas, motives, and behaviors. Corey, Corey, and Callahan (1998) found that the consensus among counselors regarding their role was to (1) promote client autonomy and (2) assist the client in making their own decisions. In essence, the internal evaluator is an organizational counselor. In this role, the evaluator seeks to empower the organization through exploring values, defining problems, gathering information, and fostering organizational change and learning based on its needs. The internal evaluator in the case study interacts with a very large family of sorts, each member with his/her own values, motivations, agendas, and objectives to foster organizational behaviors not unlike human autonomy. An evaluator who adopts the role of counselor in his/her interactions with clients enables the organization to improve itself. In the case study, the evaluator plays the role of counselor. This is indicated by the evaluator's encouragement of the group's own exploration and decision-making. Neither the counselor nor the evaluator exist to make the client's decisions, but to engage the client in an exploration of the self and the paths that could be taken.

The empowerment an evaluator provides an organization potentially parallels the relationship of therapist and client. Collaborative evaluation (O'Sullivan \& O'Sullivan, 1998) seeks to provide a participatory approach in evaluative inquiry that empowers organizational stakeholders in decision-making processes. A collaborative approach to evaluation fosters personal, team, and organizational learning and is perhaps a model of evaluation that has the potential to embrace an evaluator who takes on a counseling role. As a long-term outcome, collaborative evaluation has the potential to foster an organizational culture that embraces autonomy on the personal, team, and organizational levels. For that reason, stakeholders cite the problematic issues, develop methods for information gathering, format evaluation reports, and make recommendations for change. This approach is not dissimilar to a client-centered counselor's approach.

The origins of the modern client-centered approaches are rooted in Client-Centered Therapy (Rogers, 1951). It entails an approach to therapy that is non-directive, such that clients take the lead in illustrating and exploring problems and issues. In this approach, clients are respected individuals, each with their own morals, beliefs, and goals. Furthermore, this approach is consistent with the recent demand for consumer-driven services as well as the spirit and intent of evaluative inquiry for organizational learning (see Preskill \& Torres, 1999). Because clients, whether organizational stakeholders or seekers of healthy psychological development, 
are responsible for their own growth, learning, and decisions, they look upon the evaluator or counselor to encourage them to bring forth the issues that most concern them. For the evaluator, this guidance has potential to foster a greater influence of the evaluation process.

An essential component of all client-centered approaches is that service delivery is guided by those being served. Client-centered approaches empower the client. This empowerment may translate into the creation of an impactful process influence. There appears to be a relationship between the client-centered approach and evaluation model utility. Stufflebeam (2001) developed ratings of the strongest types of program evaluation approaches within four categories: Utility, Feasibility, Propriety, and Accuracy. Ratings for Utility were highest among three models: Utilization-Focused, Client-Centered/Responsive, and Deliberative Democratic. All of these models involve stakeholders in the process to a large degree and, interestingly, encompass a level of client-centeredness where stakeholder perceptions are valued.

\section{Closing Comments}

As mentioned earlier, benefits of the evaluation process are rarely tied to the goals of evaluation methodologies. In a conversation with Michael Quinn Patton (Coffman, 2002), process use was cited as a major breakthrough idea that has helped define and influence modern evaluation practice:

Historically, the discussion about evaluation utilization has focused on findings and on what happens with an evaluation's findings or who uses them after the evaluation is over. Utilization-focused evaluation, in contrast, says that an evaluation's use should be considered throughout the evaluation process. In line with this idea, process use focuses on the ways in which being engaged in an evaluation can have an impact on those involved. It is about how people who participate in the evaluation learn to use and apply the logic, reasoning, and values that underlie the evaluation profession (p. 10).

Preskill and Torres (2001, p. 387) describe a vision for the future of evaluation that is grounded in "a desire for evaluation to play an expanded and more productive role within organizations." Specifically, they call for "integrating the evaluation function and evaluator role within the organization [and] reconsidering traditional evaluator roles and the skills evaluators need" (p. 388). Perhaps thinking about these issues at the outset of an evaluation has the potential to foster its influence. By doing so, there will be considerable contributions to the domain of process use/influence as we promote the profession as a "sustainable learning community" (Preskill, 2000, p. 1).

Evaluators may have been hesitant to focus on process influence, perhaps due to the lack of knowledge about the ways in which one could enhance the effect. The purpose of the article was to explore some, but not all, of the roles that the evaluator could engage in to promote benefits from the evaluation process itself. Certainly, there are other roles that have the potential to affect the evaluation process influence. An evaluator should explore his/her own knowledge, skills, abilities, and personal characteristics to develop and implement appropriate roles that have the potential to foster an enhanced evaluation process influence. It is unlikely that an evaluator could succeed in the implementation of an evaluation by selecting one role. Rather, the evaluator should begin a process of reflection upon his/herself and his/her client to determine the combination of appropriate roles and strategies that appear to be most indicative of an influential evaluation process. Again, reflection of the evaluator's own personal beliefs, values, and skills may be necessary in finding the framework to fit his/her needs and 
the needs of the organization. The evaluator described throughout the case study used an eclectic approach to determine appropriate the roles and strategies. By combining elements of client-centeredness, advocacy evaluation, and organizational learning, the evaluator was able to define and implement various roles to foster an influential evaluation process.

Finally, an important caveat to taking on the role of counselor in an organization must be mentioned. Professionals who conduct counseling in areas in which they are unqualified are considered to be practicing at least unethically and, in many cases, illegally. The point here is that the evaluator should consider the organization as a living, breathing, and evolving entity (and should focus on this level). The evaluator should not "counsel" individuals within the organization; this is the work of licensed professionals. Simply, taking on the role of organizational counselor in itself creates ethical and boundary issues. For example, the evaluator has his/her own values and beliefs and should be skilled at exposing, not imposing, them. Further research on ethical and boundary issues in internal evaluation is necessary to progress within this domain.

\section{REFERENCES}

Brisolara, S. (1998). The history of participatory evaluation and current debates in the field. In E. Whitmore (Ed.), Understanding and practicing participatory evaluation. New directions for evaluation (Vol. 80). San Francisco, CA: Jossey-Bass.

Caracelli, V. J. (2000). Evaluation use at the threshold of the twenty-first century. In V. J. Caracelli \& H. Preskill (Eds.), The expanding scope of evaluation use. New directions in evaluation (Vol. 88). San Francisco, CA: Jossey-Bass.

Catania, C. A. (1992). Learning (3rd ed.). Englewood Cliffs, NJ: Prentice-Hall.

Coffman, J. (2002, Spring). A conversation with Michael Quinn Patton. The Evaluation Exchange, 8, $10-11$.

Corey, G., Corey, M., \& Callahan, P. (1998). Issues and ethics in the helping professions (5th ed.). Pacific Grove, CA: Brooks/Cole.

Harrison, O. (1997). Open space technology: A user's guide (2nd ed.). San Fransisco, CA: Berrett-Koehler.

Hatry, H., van Houten, T., Plantz, M. C., \& Greenway, M. T. (1996). Measuring program outcomes: A practical approach. Alexandria, VA: United Way.

Kirkhart, K. E. (2000). Reconceptualizing evaluation use: An integrated theory of influence. In V. J. Caracelli \& H. Preskill (Eds.), The expanding scope of evaluation use. New directions in evaluation (Vol. 88). San Francisco, CA: Jossey-Bass.

Krueger, R. A. (1998). Moderating focus groups. Thousand Oaks, CA: Sage.

O'Sullivan, R. G., \& O'Sullivan, J. M. (1998). Evaluation voices: Promoting evaluation from within programs through collaboration. Evaluation and Program Planning, 21, 21-29.

Patton, M. Q. (1997). Utilization-focused evaluation: The new century text (3rd ed.). Thousand Oaks, CA: Sage.

Preskill, H. (1994). Evaluation's role in enhancing organizational learning: A model for practice. Evaluation and Program Planning, 17, 291-297.

Preskill, H. (2000, November). The evaluation profession as a sustainable learning community. Paper presented at the American Evaluation Association Conference, Honolulu, HI.

Preskill, H., \& Caracelli, V. J. (1997). Current and developing conceptions of use: Evaluation use TIG survey results. Evaluation Practice, 18, 209-225.

Preskill, H., \& Torres, R. T. (2001). Evaluation and organizational learning: Past, present, and future. American Journal of Evaluation, 22, 387-395. 
Preskill, H., \& Torres, R. T. (1999). Evaluative inquiry for learning in organizations. Thousand Oaks, CA: Sage.

Rogers, C. R. (1951). Client-Centered Therapy. Boston, MA: Houghton Mifflin.

Scriven, M. (2001). Evaluation: Future tense. American Journal of Evaluation, 22, 301-307.

Sonnichsen, R. C. (2000). High impact internal evaluation: A practitioner's guide to evaluating and consulting inside organizations. Thousand Oaks, CA: Sage.

Stufflebeam, D. L. (2001). Evaluation models. New directions in evaluation (Vol. 89). San Francisco, CA: Jossey-Bass. 